\title{
Two- and three-dimensional numerical simulations of the clap-fling-sweep of hovering insects
}

\author{
D. Kolomenskiy ${ }^{\mathrm{a}, *}$, H.K. Moffatt ${ }^{\mathrm{b}}$, M. Farge $^{\mathrm{c}}$, K. Schneider $^{\mathrm{a}, \mathrm{d}}$ \\ a M2P2-CNRS, Universités d'Aix-Marseille, 38 rue Joliot-Curie, 13451 Marseille Cedex 20, France \\ ${ }^{\mathrm{b}}$ Department of Applied Mathematics and Theoretical Physics, University of Cambridge, Wilberforce Road, Cambridge CB3 0WA, UK \\ c LMD-IPSL-CNRS, Ecole Normale Supérieure, 24 rue Lhomond, 75231 Paris Cedex 5, France \\ ${ }^{\mathrm{d}}$ CMI, Université de Provence, 39 rue Joliot-Curie, 13453 Marseille Cedex 13, France
}

\section{A R T I C L E I N F O}

\section{Article history:}

Received 12 November 2010

Received in revised form

25 April 2011

Accepted 18 May 2011

Available online 17 June 2011

Keywords:

Flapping flight

Spanwise flow

Delayed stall

Volume penalization method

Spectral method

\begin{abstract}
A B S T R A C T
The importance of three-dimensional effects for flapping wings is addressed by means of numerical simulation. In particular, the clap-fling-sweep mechanism is examined. The flow at the beginning of the downstroke is shown to be in reasonable agreement with the two-dimensional approximation. After the wings move farther than one chord length apart, three-dimensional effects become essential. Two values of the Reynolds number are considered. At $\mathrm{Re}=128$, the spanwise flow from the wing roots to the wing tips is driven by the centrifugal forces acting on the mass of the fluid trapped in the recirculation bubble behind the wings. It removes the excess of vorticity and delays the periodic vortex shedding. At $\operatorname{Re}=1400$, vortex breakdown occurs past the outer portion of the wings, and multiple vortex filaments are shed into the wake.
\end{abstract}

(c) 2011 Elsevier Ltd. All rights reserved.

\section{Introduction}

One of the major hypotheses, that underlies many aerodynamic theories (see, e.g., Ellington, 1978) and computational studies (see, e.g., Wang, 2005) of insect hovering flight, states that the near flow can be sufficiently well described within the two-dimensional approximation. This viewpoint is classical. It is very appealing since it greatly simplifies the modelling and, together with other assumptions, can even lead to an analytical description of the flow. The twodimensional approximation is well justified for wings of large aspect ratio, but for real insect wings this parameter varies between 1 and 6 (for a single wing). It is therefore important to assess the significance of three-dimensionality of the flow and the validity of the two-dimensional approximation.

Firstly, the length of the wings is finite. The pressure difference between the lower and the upper surfaces forces the air to flow around the tips. As a result, less lift is generated near the wing ends. In addition, tip vortices shed into the flow modify the forces generated at all spanwise positions of the wing.

Secondly, the wings rotate, and this makes a big difference, as compared to the same wings in rectilinear translational motion (see, e.g., Sane, 2003). Insect wings typically operate at large angles of attack, where the flow separates from sharp leading edges, and strong leading-edge vortices are formed. As long as they remain near the wings, depression in their cores leads to larger lift.

The dynamics of the leading-edge vortices is strongly influenced by the three-dimensional nature of the flow. As first observed by Maxworthy (1979) in his experiments with a dynamically scaled model, these vortices follow the wings during the entire downstroke. This feature makes a striking contrast to periodic vortex shedding that occurs in the twodimensional motion.

\footnotetext{
* Corresponding author.

E-mail address: dkolom@gmail.com (D. Kolomenskiy).
} 
This 'delayed stall' appears to be very common for all flying insects. For instance, stably attached leading-edge vortices were observed in experiments with hawkmoths (Ellington et al., 1996), butterflies (Srygley and Thomas, 2002), dynamically scaled robotic flies (Birch and Dickinson, 2001; Lehmann et al., 2005) and also in numerical simulations (e.g., Lan and Sun, 2001; Liu and Ellington, 1998). This behaviour of the leading-edge vortex is associated with the air flow in spanwise direction along the wing. Maxworthy (2007) made an estimate of the spanwise velocity assuming that the vortex has a conical tornado-like shape. Birch et al. (2004) observed experimentally, and Aono et al. (2008) confirmed numerically, that the structure of the leading-edge vortex significantly differs between the values of the Reynolds number Re of order 100 and of order 1000. Lentink and Dickinson (2009) argued that stable attachment of these vortices occurs when the Rossby number (identical to the aspect ratio of a single wing) is less than 3. However, a quantitative description of how the spanwise flow affects vorticity dynamics is still incomplete.

The purpose of this work is to quantify three-dimensional effects in the flow due to the clap-fling-sweep mechanism (Lighthill, 1973; Weis-Fogh, 1973). In particular, we assess the validity of the two-dimensional approximation, as anticipated in our previous study Kolomenskiy et al. This unsteady aerodynamic mechanism is used by some insects when they need more lift at the beginning of their wing stroke. When the stroke amplitude is large, the wings eventually touch with their surfaces. While the wings pronate, their trailing edges the remain together, impeding the air flow between them. Much stronger vortices are therefore generated at the leading edges, compared to normal hovering when the wings do not clap, as first suggested by Maxworthy (1979). Later on, several authors explored this by numerical simulations (Haussling, 1979; Kolomenskiy et al., 2010; Kolomenskiy et al.; Miller and Peskin, 2005; Sohn and Wu, 1987; Sun and Yu, 2003), yet the models were two-dimensional and neglected some important phenomena mentioned earlier in this introduction. Three-dimensional numerical simulations were performed by Sun and Yu (2006) who restricted their attention to $R e=15$, which is low enough for the leading-edge vortices to be stable even in a two-dimensional flow. Herein we consider $\mathrm{Re}=128$ corresponding to fruit flies and $\mathrm{Re}=1400$ more typical of butterflies.

The remainder of this paper is organized as follows. In Section 2, we give a short description of the physical model and the numerical method. Section 3 explains the geometry and kinematics of the flapping wings model. The results are presented in Section 4, and the conclusions are drawn in Section 5.

\section{Governing equations and numerical discretization}

We consider rigid wings moving in a viscous incompressible fluid. The no-slip boundary condition at the solid boundary is modelled using the volume penalization method (Angot et al., 1999).

The three-dimensional solver employs the rotational formulation of the penalized Navier-Stokes equation,

$$
\frac{\partial \boldsymbol{u}_{\eta}}{\partial t}+\omega_{\eta} \times \boldsymbol{u}_{\eta}+\nabla \Pi_{\eta}-v \nabla^{2} \boldsymbol{u}_{\eta}+\frac{\chi_{\Omega}}{\eta}\left(\boldsymbol{u}_{\eta}-\boldsymbol{u}_{s}\right)=0,
$$

with the vorticity $\omega_{\eta}=\nabla \times \boldsymbol{u}_{\eta}$ and the modified pressure $\Pi_{\eta}=p_{\eta}+u_{\eta}^{2} / 2$. The incompressibility condition $\nabla \cdot \mathbf{u}_{\eta}=0$ yields

$$
-\nabla^{2} \Pi_{\eta}=\nabla \cdot\left(\omega_{\eta} \times \boldsymbol{u}_{\eta}+\frac{\chi_{\Omega}}{\eta}\left(\boldsymbol{u}_{\eta}-\boldsymbol{u}_{s}\right)\right) .
$$

In the above equations the unknowns are the velocity $\boldsymbol{u}_{\eta}$ and the pressure $p_{\eta}$. The parameter $v$ is the kinematic viscosity, while the density of the fluid $\rho$ is normalized to unity. The mask function $\chi_{\Omega}$ describes the geometry of the wings, it equals 1 inside of the solid and 0 inside of the fluid. The vector field $\boldsymbol{u}_{s}$ is the local velocity of the solid medium. $\eta$ is the penalization parameter. By solving Eq. (2) the divergent part of the nonlinear and the penalization terms is removed.

Eqs. (1) and (2) are solved using a classical Fourier pseudo-spectral method. The spatial computational domain is a rectangular box with periodic boundary conditions imposed on all its faces. It is discretized with a uniform Cartesian grid. The time integration is exact for the viscous term and the adaptive second order Adams-Bashforth scheme is used for the nonlinear term. Our parallel implementation of the code employs the P3DFFT fast Fourier transform package. ${ }^{1}$

The two-dimensional solver is based on the vorticity-stream function formulation of the Navier-Stokes equation. The numerical method is essentially the same. Its details were reported earlier by Kolomenskiy and Schneider (2009).

\section{Flow configuration}

Unlike in some other computational studies of insect flight, the present numerical experiments do not aim at reproducing subtleties of flapping wings of real insects, but rather focus on the essential features explained in the introduction.

A model wing, shown in Fig. 1(a), has a very simple shape: its planform is a circular sector and its cross-section is rectangular. The linear dependence of the chord length $c$ on the radius $r$, inspired by the experimental setup of Maxworthy (1979), is convenient for analysis of the spanwise distribution of different aerodynamic quantities. The wing dimensions are chosen such that the chord length equals $c^{*}=1$ at the radius $r^{*}=3$. The full wing length from root to tip equals $R=4$.

\footnotetext{
${ }^{1}$ p3dfft.googlecode.com
} 

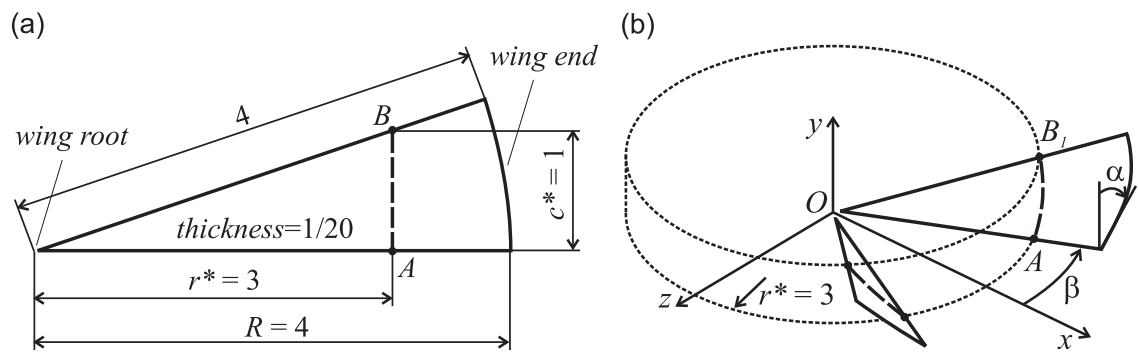

Fig. 1. Sketch of one wing (a) and the assembly of two flapping wings (b).

(a)

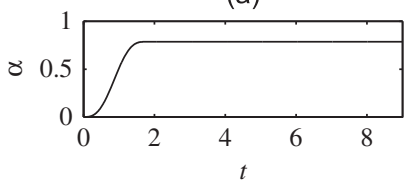

(c)

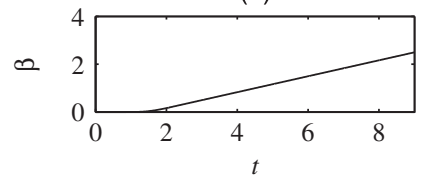

(b)

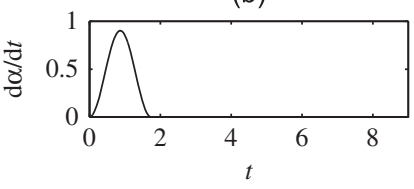

(d)

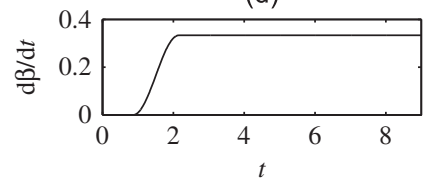

Fig. 2. Wing kinematics: time evolution of the angles $\alpha$ and $\beta$ and their derivatives.

Fig. 1(b) shows an assembly of the two wings. Each of them rotates with respect to a hinge point situated at the vertex of the sector's sharp angle, in the mid-thickness plane. The distance between the hinges equals $d_{0}=0.1$.

The kinematics of the wings is depicted in Fig. 2. Initially the trailing edges are aligned with the $O x$ line, and the wings touch with their upper surfaces-'clap'. The wings start rotating first about the trailing edges, the angle $\alpha$ increases up to $45^{\circ}$ - 'fling'. Then the wings rotate about the $y$ axis-'sweep'. The two wings remain symmetrical with respect to the $x y$ plane. The angular velocities are imposed as

$$
\dot{\alpha}=\frac{\Delta \theta}{\Delta t_{\text {rot }}}\left[1.0-\cos \left(\frac{2 \pi}{\Delta t_{\text {rot }}} t\right)\right] \text { for } t<\Delta t_{\text {rot }}
$$

and

$$
\begin{aligned}
& \dot{\beta}=\frac{U^{*}}{2 r^{*}}\left[1.0+\cos \left(\pi+\pi \frac{t-t_{\text {accel }}}{\Delta t_{\text {accel }}}\right)\right] \text { for } t_{\text {accel }} \leq t<t_{\text {accel }}+\Delta t_{\text {accel }}, \\
& \dot{\beta}=\frac{U^{*}}{r^{*}} \text { for } t \geq t_{\text {accel }}+\Delta t_{\text {accel }},
\end{aligned}
$$

where $\Delta \theta=\pi / 4, \Delta t_{\text {rot }}=1.74, \Delta t_{\text {accel }}=1.3, t_{\text {accel }}=0.86, U^{*}=1$.

Two values of the Reynolds number are considered, $\operatorname{Re}=U^{*} C^{*} / v=128$ and 1400 . This corresponds to $v=0.0078125$ and 0.00071429 , respectively. The penalization parameter is $\eta=10^{-3}$ and $\eta=5 \times 10^{-3}$, respectively. The periodic box size is $L_{x} \times L_{y} \times L_{z}=11^{3}$, and the number of grid points is $N_{x} \times N_{y} \times N_{z}=1024^{3}$.

In addition, two-dimensional numerical simulations are performed, where the solid body motion corresponds to that of the wing sections at $r^{*}$ on the cylindrical surface sketched in Fig. 1(b). The domain size in that case is $L_{x} \times L_{y}=2 \pi r^{*} \times 11$ and the number of grid points is either $N_{x} \times N_{y}=1754 \times 1024$ or $3508 \times 2048$. Note that all physical quantities are presented in a dimensionless form. The distances are normalized by the chord length at three quarters from the root, shown in Fig. 1, and the time scale is given by the inverse of the terminal angular velocity.

\section{Results and discussion}

\subsection{Reynolds number $\mathrm{Re}=128$}

Fig. 3 depicts the absolute value of the vorticity at three consequent time instants. Red color corresponds to high magnitude of vorticity, and it is mainly seen in the vortices attached to the wing edges. Blue color indicates lower vorticity, and it is observed in the free vortex wake. During fling, at $t=1.2$, very strong leading-edge vortices are seen. Equally strong vortices are generated at the wing tips, but the flux between them is only about $15 \%$ of the flux between the leading edges. This vorticity results from the air flow into the opening space between the wings past its sharp edges. Also note a vortex 


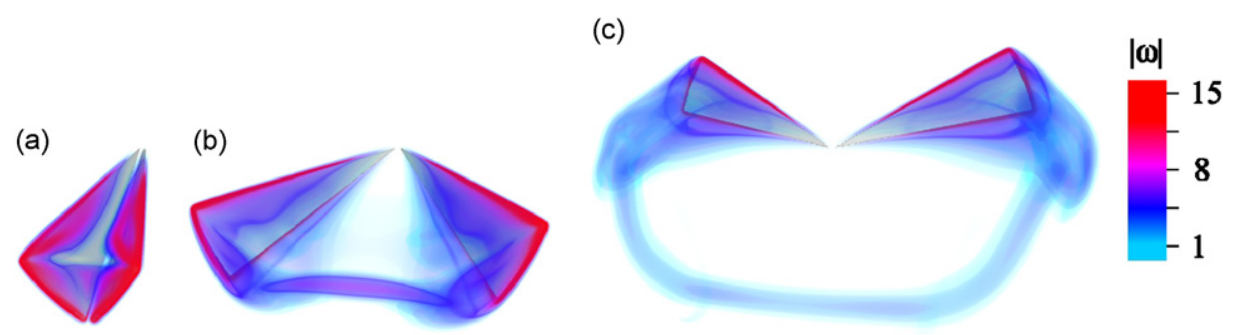

Fig. 3. Vorticity magnitude at time instants (a) $t=1.2$, (b) $t=3.2$, (c) $t=7.2$ at the Reynolds number Re $=128$. (For interpretation of the references to color in this figure legend, the reader is referred to the web version of this article.)

(a)

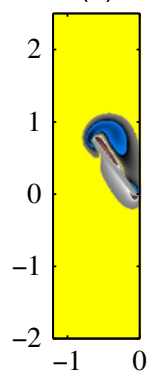

(b)

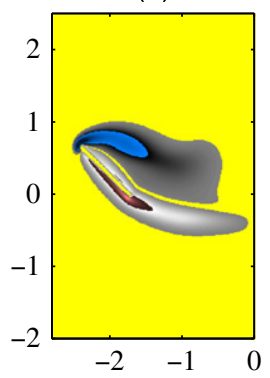

(c)

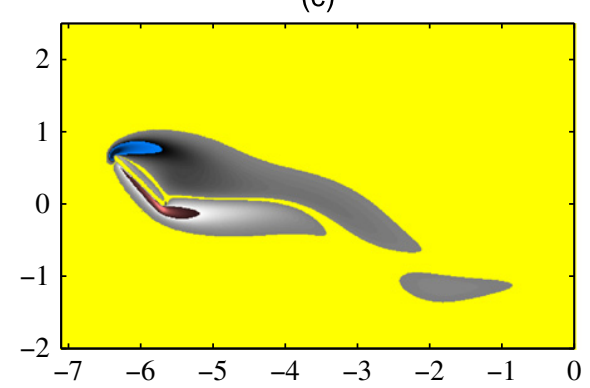

$\omega_{\mathrm{r}}$

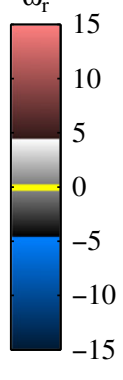

Fig. 4. Radial component of the vorticity probed on a cylinder surface $r=0.75 R$. Time instants are (a) $t=1.2$, (b) $t=3.2$, (c) $t=7.2$.

(a)

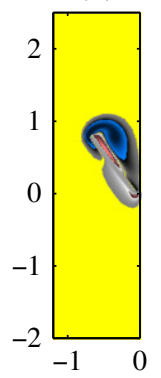

(b)

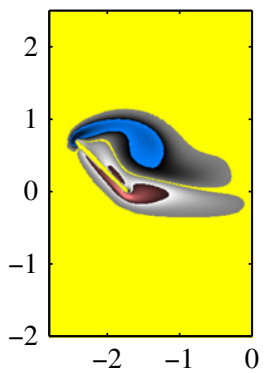

(c)

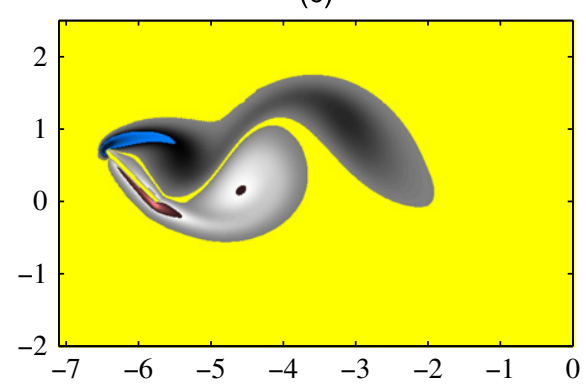

$\omega$

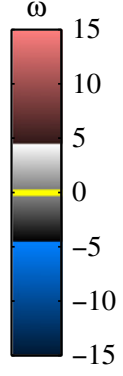

Fig. 5. Vorticity field in the two-dimensional numerical simulation. Time instants are (a) $t=1.2$, (b) $t=3.2$, (c) $t=7.2$.

which reconnects the wing ends. It forms a horseshoe shape at later times, $t=3.2$ and 7.2. At $t=7.2$ the vortices shed from the wing ends resemble hairpins typical for flows past three-dimensional bluff bodies like spheres or disks.

It is straightforward to compare these results with those predicted within the two-dimensional approximation. In Figs. 4 and 5, a cylindrical section of the three-dimensional field (radial component only) is compared with the two-dimensional result. At the beginning, $t=1.2$, the vorticity fields look similar in both cases. The two-dimensional free vortices are slightly stronger. The discrepancy becomes large at $t=3.2$.

When the wings move far apart, $t=7.2$, the dynamics of the free vortices in the two cases becomes drastically different. In the two-dimensional simulation, the leading- and the trailing-edge vortices grow in strength until they are subsequently shed, and the process would repeat periodically if the wings continued to move further. This agrees with previous results by Miller and Peskin (2005). The three-dimensional vortices are less intense, but they remain attached and their strength remains constant in time after $t \approx 4$. The near flow field of a wing is approximately steady in a moving reference frame. The horseshoe vortex is visible in the far field.

The lift coefficient per wing is calculated using the following formula:

$$
c_{L}=\frac{2 L}{\rho U^{* 2} A},
$$

where $L$ is the vertical component of the aerodynamic force, called here 'lift' for short, and $A$ is the wing area, $A=0.5 R^{2} \arctan c^{*} / r^{*} \approx 2.57$. Fig. 6 depicts the lift coefficient (5) as a function of time, compared with the same quantity 


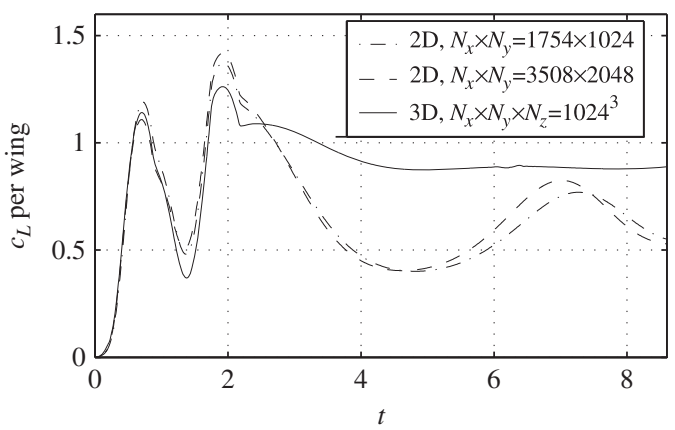

Fig. 6. Time evolution of the lift coefficient. Comparison of the result from a three-dimensional numerical simulation with two-dimensional estimates for two different grid sizes.

(a)

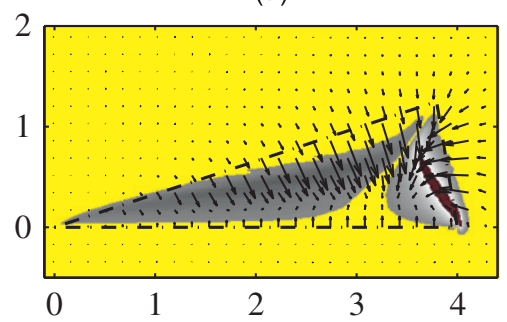

(b)

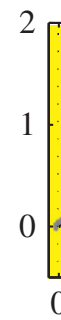

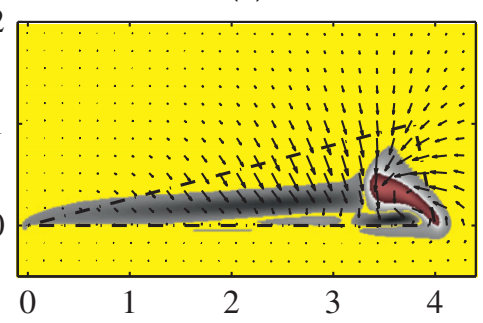

$\omega_{\theta}$

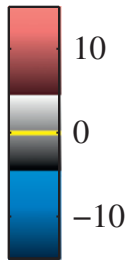

Fig. 7. Velocity (arrows) and azimuthal vorticity (colour) in the symmetry plane Oxy during fling at (a) $t=0.6$, (b) $t=12$. (For interpretation of the references to color in this figure legend, the reader is referred to the web version of this article.)

estimated using the two-dimensional simulations,

$$
c_{L} \approx \int_{0}^{R_{\text {eff }}} c_{L \mathrm{AR}=\infty} \frac{U(r)^{2}}{U^{* 2}} \frac{1}{A} c(r) \mathrm{d} r .
$$

To derive this formula, the lift coefficient of a planar section at distance $r$ is approximated by the lift coefficient of the reference section at distance $r^{*}, c_{L A R}=\infty=2 L_{\mathrm{AR}=\infty} / \rho U^{* 2} c^{*}$, scaled with the chord length $c(r)=c^{*} r / r^{*}$ and the velocity $U(r)=U^{*} r / r^{*}$. The sectional lift force $L_{\mathrm{AR}=\infty}$ is a result of the two-dimensional numerical simulation. Integration is carried up to the efficient wing length $R_{e f f}=0.88 R$ obtained from the spanwise distribution of circulation described below. A comparison between the two-dimensional calculations made with two different grids suggests that the accuracy is enough for at least a qualitative analysis.

During fling, $c_{L}$ is mainly driven by the time evolution of $\dot{\alpha}^{2}$. It grows rapidly and then drops, reaching its minimum at $t=1.3$, when both velocities $\dot{\alpha}$ and $\dot{\beta}$ are quite small. Another peak follows at $t=1.9$ as an optimum between $\dot{\beta}^{2}$ increasing and $\ddot{\beta}$ decreasing in time. This behaviour agrees well with two-dimensional numerical simulations of Miller and Peskin (2005), Sun and Yu (2003) and three-dimensional experiments of Lehmann et al. (2005). The two-dimensional approximation remains adequate during fling and the beginning of sweep, until $t \approx 2$, when separation of the trailing edges exceeds one chord length. The latter discrepancy is a direct consequence of the delayed stall. The two-dimensional estimate is oscillating, its amplitude is as large as its mean value. The value resulting from the three-dimensional simulation saturates after $t=4$, and it is larger.

A comparison between robotic wing experiments and two-dimensional computations of normal hovering was earlier performed by Wang et al. (2004). They proved the two-dimensional approximation to be sufficient to predict aerodynamic forces when the stroke amplitude is such that the wing travels between 3 and 5 chord lengths. But the clap-fling-sweep implies a larger stroke amplitude (6 to 8 chord lengths), therefore the two-dimensional vortex shedding occurs well before the end of the downstroke.

Obviously, the key feature absent in the two-dimensional approximation is the spanwise flow. Knowing that the twodimensional approximation is good enough to predict the lift coefficient during fling, it is instructive to compare the spanwise and the in-plane components of the velocity.

Fig. 7 presents this comparison for two different time instants, at the beginning and at the end of fling. Arrows visualize the velocity in the symmetry plane $O x y$ (i.e., a side view), where the radial component is maximized and the azimuthal component vanishes due to symmetry. In Fig. 7, colour depicts the azimuthal component of the vorticity. At $t=0.6$ the velocity is perpendicular to the edges, therefore its radial component is relatively small between $r=1.5$ and $r=3.5$. Also note some inflow through a small gap between the trailing edges. At $t=1.2$ the spanwise flow increases, and only between $r=2.5$ and $r=3.5$ the flow remains effectively two-dimensional (meanwhile this part of the wing generates most lift). 
(a)

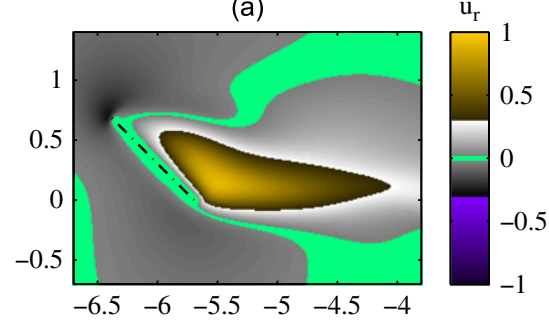

(b)

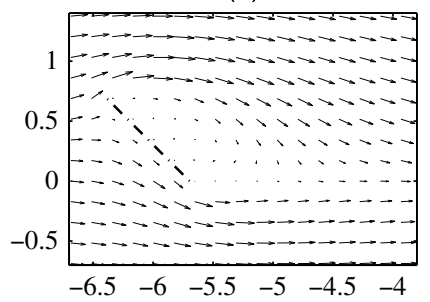

Fig. 8. Radial (a) and tangential (b) components of the velocity in the cross-section $r=0.75 R$ at time $t=7.2$. (For interpretation of the references to color in this figure legend, the reader is referred to the web version of this article.)

(a)

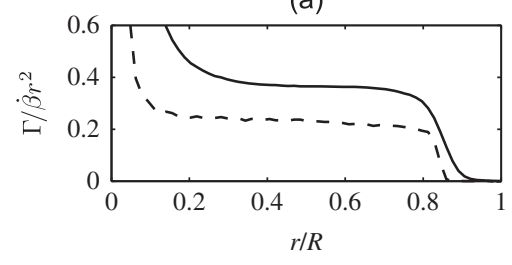

(b)

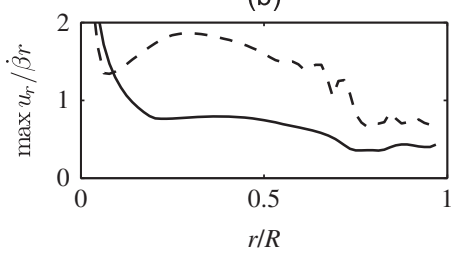

Fig. 9. Normalized circulation (a) and maximum radial velocity (b) versus the dimensionless distance from the wing root at time $t=3.2$. The solid lines correspond to the case $\operatorname{Re}=128$ and the dashed lines to the case $\operatorname{Re}=1400$.

Near the wing ends, at $r>3.5$, the flow is essentially in the spanwise direction. The strong vertical gradient of the velocity creates a vortex (shown in red) which then rolls up and advects inwards.

When the wings move apart, the radial velocity increases and its maximum becomes of order unity, as displayed in Fig. 8(a). The strong spanwise flow from root to tip occupies a large domain behind the wing, but this is not where the vorticity is strong (cf. Fig. 4(b)). The vorticity is the strongest near the sharp edges, where, surprisingly, the spanwise flow is in the opposite direction, from tip to root. These features can be observed in experimental measurements by Birch and Dickinson (2001) and in the numerical simulation by Aono et al. (2008).

The reason for the strong spanwise flow from root to tip behind the upper surface is evident from the velocity field relative to the wing, shown in Fig. 8 (b). It reveals a recirculation bubble, and centrifugal forces acting on it generate the spanwise flow. To explain why the maximum spanwise flow from root to tip occurs outside the vortex cores, let us examine the radial component of the vorticity equation:

$$
\frac{\mathrm{D} \omega_{r}}{\mathrm{D} t}-\boldsymbol{\omega} \cdot \nabla u_{r}=0
$$

The viscous term is neglected for simplicity. D/Dt denotes the material derivative. Let us follow a Lagrangian fluid particle which is at time $t_{0}$ located at a point such that its radial coordinate is $r_{0}$ and the radial velocity is maximized (centre of the red colour area in Fig. 8(a), for instance). At that point both derivatives $\partial u_{r} / \partial \theta$ and $\partial u_{r} / \partial y$ are zero. Eq. (7) thus becomes

$$
\frac{\mathrm{D} \omega_{r}}{\mathrm{D} t}-\omega_{r} \frac{\partial u_{r}}{\partial r}=0
$$

The radial gradient $\partial u_{r} / \partial r$ is positive and varies little with $r$ (see Fig. 9). Moreover, it remains approximately constant in time, since the particle is trapped in the recirculation bubble behind the wing. In these conditions the vorticity carried by the fluid particle under consideration would increase exponentially in time, and the only solution compatible with the steady flow is zero. This is slightly modified by the action of viscous diffusion and other imperfections.

Some flow in the opposite direction, from tip to root, occurs near the lower surface because of the continuity. It peaks in front of the leading edge. Parametric studies (not shown here) indicate that this peak diminishes with smaller wing thickness.

Fig. 9 (solid lines) shows how the circulation and the spanwise velocity vary in the spanwise direction. The circulation at distance $r$ is computed by integrating the radial vorticity throughout the right half of the corresponding cylindrical section. The maximum radial velocity is sought in the same domain. The circulation is proportional to $r^{2}$, and the maximum spanwise velocity is increasing linearly with $r$, except that near the root there are low Reynolds number effects, and near the tips, at $r \approx 0.88 R$, the circulation drops to zero. The latter value is used to define the upper integration limit in (6).

\subsection{Reynolds number $\operatorname{Re}=1400$}

Experiments of Birch et al. (2004) showed that the flow at $\operatorname{Re}=1400$ differs qualitatively from what is observed at Re of order 100. Our numerical simulations confirm this fact, but some details are new and some further insights are given. 
(a)

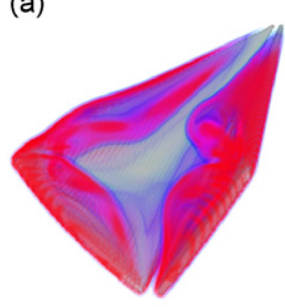

(b)

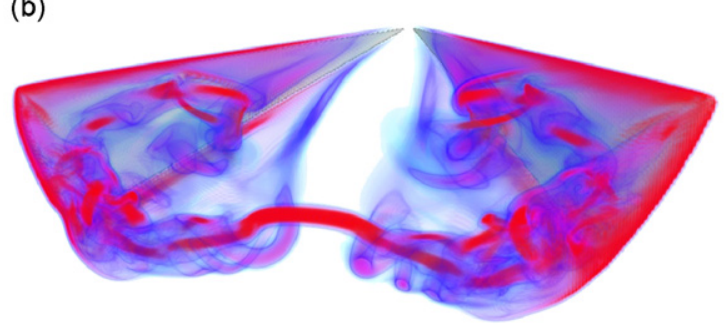

$|\omega|$

$-30$

15.5

1

Fig. 10. Vorticity magnitude at time instants (a) $t=1.2$, (b) $t=3.2$ at the Reynolds number $\operatorname{Re}=1400$.

(a)

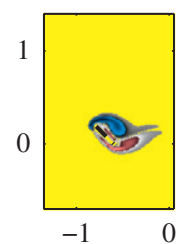

$\omega$

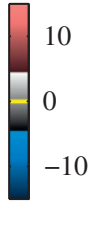

(b)

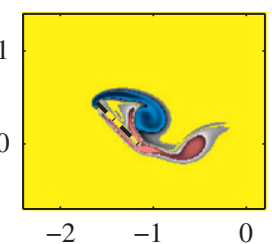

$\omega_{\mathrm{r}}$

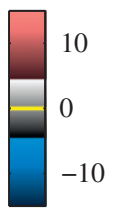

(c)

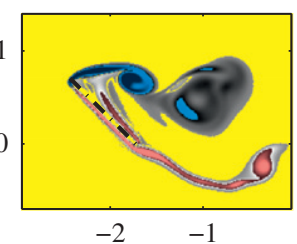

$\omega_{\mathrm{r}}$

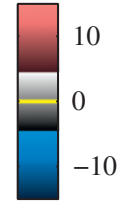

Fig. 11. Radial component of the vorticity at time $t=3.2$. Three radial positions are shown, (a) $r=0.25 R$, (b) $0.5 R$, (c) $0.75 R$.

(a)

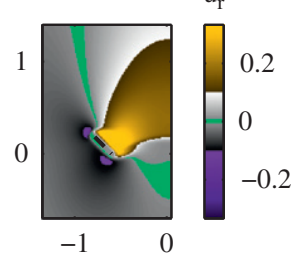

(b)

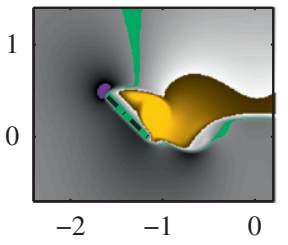

$\mathrm{u}_{\mathrm{r}}$

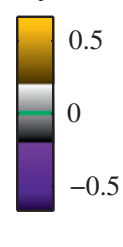

(c)

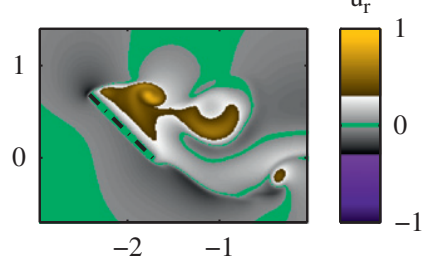

Fig. 12. Radial component of the velocity at time $t=3.2$. Three radial positions are shown, (a) $r=0.25 R$, (b) $0.5 R$, (c) $0.75 R$.

Fig. 10 shows the vorticity field at two time instants. At the end of fling, $t=1.2$, the vortex pattern is essentially the same as observed at the lower Reynolds number, but the structure of vortex cores is different: thin vorticity layers generated at the edges roll up into conical vortices. The vorticity peaks inside their cores and near the wing edges. The conical vortices are slightly distorted near the corner at the wing ends. This perturbation grows, and at $t=3.2$ there are two distinct spanwise zones: near the wing root the vortex is stable and follows the wing; near the wing ends the vortex core breaks down and multiple filaments are shed into the wake. The delayed stall therefore takes place only near the wing root. The structure of the horseshoe vortex also differs from the lower Reynolds number case. It is more compact and intense. In addition, as the wings move apart, the trailing-edge vortex separates and spirals around it.

Fig. 11 displays the radial component of the vorticity at $t=3.2$ at three cylindrical sections. The flow field at $r=0.25 R$ exhibits a laminar pattern with one leading- and one trailing-edge vortex following the wing. At $r=0.5 R$ the leading-edge vortex is less diffuse, and the trailing-edge vortex is shed. Visualization at $r=0.75 R$ reveals two subsequently shed leadingedge vortices, the first of them bursts into multiple pieces.

The radial velocity is shown in Fig. 12. At $r=0.25 R$ it is positive in a large area past the wing, and slightly negative in front of it. This is similar to the lower-Reynolds number case, but the magnitude is now twice as large. At $r=0.5 R$ the velocity is very large in the vortex core. At $r=0.75 R$ local peaks are seen in the detached vortex filaments.

The dashed line in Fig. 9(a) depicts spanwise distribution of the circulation at $t=3.2$. Despite the drastic change in the vortex structure at $r=0.5 R$, on both sides $\Gamma=0.22 \dot{\beta} r^{2}$ is a good approximation (except very near the wing ends). The distribution of the maximum radial velocity Fig. 9(b) (dashed line) reveals the two zones: at $r<0.5 R$ it varies gradually with $r$, mounting to $u_{r} / \dot{\beta} r=1.9$, while at $r>0.5 R$ it oscillates and drops to much smaller values.

The spanwise flow from root to tip is driven by two motives: the centrifugal force and the pressure gradient inherent to vortices. For the conical vortices at $r<0.5 R$ the two contributions are of the same order of magnitude, as explained by Maxworthy (2007). This is the reason why the spanwise velocity is so large, compared to the lower-Reynolds number case where, presumably, only the centrifugal force matters. 


\section{Conclusions}

Two- and three-dimensional numerical simulations of the Lighthill-Weis-Fogh mechanism indicate that during fling, the two-dimensional approximation is well justified; during sweep, three-dimensionality of the flow leads to the delayed stall.

The three-dimensional flow fields at $\mathrm{Re}=128$ and $\mathrm{Re}=1400$ differ in many features. At $\operatorname{Re}=128$ the strongest vorticity is found at the wing edges, and the maximum spanwise flow from root to tip is in the recirculation bubble past the wings. Some inverse spanwise flow occurs below the lower surfaces and it peaks near the leading edges. The near flow field is steady in the reference frame moving with the wing, provided the wing motion is steady (like during sweep).

At $\operatorname{Re}=1400$ the vorticity sheets shed from the leading edges roll up into conical vortices. They burst near the outer halves of the wings. Radial velocity is large inside the vortex cores, and particularly inside the inner portions of the leading-edge vortices where it is double the reference speed of the wing section.

Scaling of the circulation like $\dot{\beta} r^{2}$ and radial velocity like $\dot{\beta} r$ suggests that similarity considerations might be helpful in a further analysis.

\section{Acknowledgments}

DK thanks Tony Maxworthy and Jean-Yves Andro for useful discussions. DK and KS acknowledge financial support from the Deutsch-Französische Hochschule, Project 'S-GRK-ED-04-05'. DK gratefully acknowledges support from the David Crighton Fellowship. Numerical simulations were performed using HPC resources of IDRIS, Paris, Project 81664. Imagery in Figs. 3 and 10 was produced with VAPOR (www.vapor.ucar.edu), a product of the National Center for Atmospheric Research (Clyne et al., 2007).

\section{References}

Angot, P., Bruneau, C.H., Fabrie, P., 1999. A penalisation method to take into account obstacles in viscous flows. Numerische Mathematik 81, 497-520. Aono, H., Liang, F., Liu, H., 2008. Near- and far-field aerodynamics in insect hovering flight: an integrated computational study. Journal of Experimental Biology 211, 239-257.

Birch, J.M., Dickinson, M.H., 2001. Spanwise flow and the attachment of the leading-edge vortex on insect wings. Nature 412, 729-733.

Birch, J.M., Dickson, W.B., Dickinson, M.H., 2004. Force production and flow structure of the leading edge vortex on flapping wings at high and low Reynolds numbers. Journal of Experimental Biology 207, 1063-1072.

Clyne, J., Mininni, P., Norton, A., Rast, M., 2007. Interactive desktop analysis of high resolution simulations: application to turbulent plume dynamics and current sheet formation. New Journal of Physics 9, 301.

Ellington, C.P., 1978. The aerodynamics of normal hovering flight: three approaches. In: Comparative Physiology-Water, Ions and Fluid Mechanics. Cambridge University Press, pp. 327-345.

Ellington, C.P., van den Berg, C., Willmott, A.P., Thomas, A.L.R., 1996. Leading-edge vortices in insect flight. Nature 384, 626-630.

Haussling, H.J., 1979. Boundary-fitted coordinates for accurate numerical solution of multibody flow problems. Journal of Computational Physics 30, 107-124.

Kolomenskiy, D., Moffatt, H.K., Farge, M., Schneider, K. The Lighthill-Weis-Fogh clap-fling-sweep mechanism revisited. Journal of Fluid Mechanics, in press. doi:10.1017/jfm.2011.83.

Kolomenskiy, D., Moffatt, H.K., Farge, M., Schneider, K., 2010. Vorticity generation during the clap-fling-sweep of some hovering insects. Theoretical and Computational Fluid Dynamics 24, 209-215, doi:10.1007/s00162-009-0137-2.

Kolomenskiy, D., Schneider, K., 2009. A Fourier spectral method for the Navier-Stokes equations with volume penalization for moving solid obstacles. Journal of Computational Physics 228, 5687-5709.

Lan, S.L., Sun, M., 2001. Aerodynamic properties of a wing performing unsteady rotational motions at low Reynolds number. Acta Mechanica 149, 135-147.

Lehmann, F.O., Sane, S.P., Dickinson, M., 2005. The aerodynamic effects of wing-wing interaction in flapping insect wings. Journal of Experimental Biology 208, 3075-3092.

Lentink, D., Dickinson, M.H., 2009. Rotational accelerations stabilize leading edge vortices on revolving fly wings. Journal of Experimental Biology 212, 2705-2719.

Lighthill, M.J., 1973. On the Weis-Fogh mechanism of lift generation. Journal of Fluid Mechanics 60, 1-17.

Liu, H., Ellington, C.P., Kawachi, K., van den Berg, C., Willmott, A.P., 1998. A computational fluid dynamic study of hawkmoth hovering. Journal of Experimental Biology 201, 461-477.

Maxworthy, T., 1979. Experiments on the Weis-Fogh mechanism of lift generation by insects in hovering flight. Part 1. Dynamics of the 'fling'. Journal of Fluid Mechanics 93, 47-63.

Maxworthy, T., 2007. The formation and maintenance of a leading-edge vortex during the forward motion of animal wing. Journal of Fluid Mechanics 587, 471-475

Miller, L.A., Peskin, C.S., 2005. A computational fluid dynamics of ‘clap and fling' in the smallest insects. Journal of Experimental Biology 208 , $195-212$.

Sane, S.P., 2003. The aerodynamics of insect flight. Journal of Experimental Biology 206, 4191-4208.

Sohn, M.H., Wu, J.C., 1987. A numerical study of the Weis-Fogh mechanism. AIAA Paper no. 238.

Srygley, R.B., Thomas, A.L.R., 2002. Unconventional lift-generating mechanisms in free-flying butterflies. Nature 420, 660-664.

Sun, M., Yu, X., 2003. Flows around two airfoils performing fling and subsequent translation and translation and subsequent clap. Acta Mechanica Sinica $19,103-117$.

Sun, M., Yu, X., 2006. Aerodynamic force generation in hovering flight in a tiny insect. AIAA Journal 44, 1532-1540.

Wang, Z.J., 2005. Dissecting insect flight. Annual Review of Fluid Mechanics 37, 183-210.

Wang, Z.J., Birch, J.M., Dickinson, M.H., 2004. Unsteady forces and flows in low Reynolds number hovering flight: two-dimensional computations vs robotic wing experiments. Journal of Experimental Biology 207, 449-460.

Weis-Fogh, T., 1973. Quick estimates of flight fitness in hovering animals, including novel mechanisms for lift production. Journal of Experimental Biology 59, 169-230. 